\title{
Equivalência semântica e confiabilidade da versão em português da Bergen Facebook Addiction Scale
}

\author{
Semantic equivalence and confiability of the Portuguese \\ version of the Bergen Facebook Addiction Scale \\ Hugo Rafael de Souza e Silva', Kelsy Catherina Nema Areco², Paulo Bandiera-Paiva², \\ Pauliana Valéria Machado Galvão3 , Anália Nusya de Medeiros Garcia', Dartiu Xavier da Silveira?
}

\section{Palavras-chave \\ Comparação transcultural, internet, escalas, estudos de validação.}

\section{RESUMO}

Objetivo: Avaliar a equivalência semântica e a confiabilidade da Bergen Facebook Addiction Scale para o português (Brasil). Métodos: $O$ processo consistiu em cinco passos: tradução; retradução; revisão técnica e avaliação da equivalência semântica por profissionais; avaliação do instrumento por compreensão verbal por uma amostra de profissionais $(n=10)$ e de estudantes ( $n=37)$; análise de consistência interna e estabilidade por meio do coeficiente de Cronbach e coeficiente de correlação intraclasse (CCI) respectivamente, em uma amostra de 359 estudantes de graduação. Resultados: $\mathrm{O}$ instrumento apresentou no fim um excelente nível de compreensão verbal pela população-alvo, alfa de Cronbach de 0,92 e CCl de 0,81. Conclusão: A versão adaptada do instrumento para o uso em nosso ambiente resultou em um instrumento equivalente do ponto de visão de equivalência semântica, assegurando a transferência do significado geral e referencial, mantendo níveis satisfatórios de confiabilidade.

\section{ABSTRACT}

Objective: To evaluate the semantic equivalence and confiability of the Bergen Facebook Addiction Scale into Portuguese (Brazil). Methods: The process consisted of five steps: translation; back-translation; technical review and evaluation of semantic equivalence by professionals; evaluation instrument as verbal comprehension by a professional sample $(n=10)$ and students ( $n=37$ ); analysis of internal consistency and stability through Cronbach coefficient and intraclass correlation coefficient (ICC) respectively, in a sample of 359 undergraduate students. Results: The instrument presented at the end an excellent level of verbal comprehension by the target population, Cronbach's alpha of 0.92 and ICC of 0.81 . Conclusion: The adapted version of the instrument for use in our environment resulted in an equivalent instrument from the point of view of semantic equivalence, ensuring the transfer of general and referential meaning, maintaining satisfactory levels of reliability.

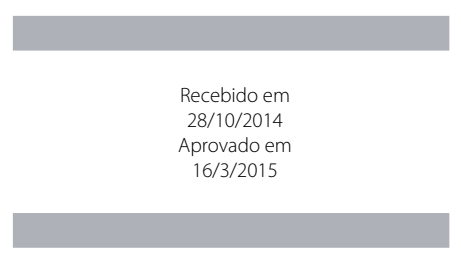

DOI: 10.1590/0047-2085000000052
1 Universidade Federal de São Paulo (Unifesp), Escola Paulista de Medicina (EPM), Departamento de Psiquiatria e Psicologia Médica. 2 Unifesp-EPM, Departamento de Informática em Saúde, Hospital São Paulo. 3 Escola Nacional de Saúde Pública Sergio Arouca, Fundação Oswaldo Cruz. 4 Universidade de Pernambuco (UPE), Instituto de Ciências Biológicas (ICB).

Endereço para correspondência: Pauliana Valéria Machado Galvão Escola Nacional de Saúde Pública Sergio Arouca, Fundação Oswaldo Cruz Rua Leopoldo Bulhões, 1480, Manguinhos 21041-210 - Rio de Janeiro, RJ, Brasil

E-mails:mscpauliana@hotmail.com.br; cons.acad@outlook.com 


\section{INTRODUÇÃO}

O Facebook é um site de rede social (RS) criado em 2004', e desde então seu número de usuários cresce exponencialmente, tornando-se o site de RS mais popular em todo o mundo ${ }^{2}$. As estimativas são de que existam 1,19 bilhão de usuários cadastrados e 728 milhões de conexões diárias. 0 Brasil possui a terceira posição em número total de usuários (76 milhões), ficando atrás apenas dos Estados Unidos (EUA) e da Índia 3 .4.

As estatísticas relativas ao uso do Facebook têm revelado que alguns indivíduos podem fazer uso excessivo de suas funcionalidades, permanecendo conectados on-line tempo suficiente para prejudicar suas atividades diárias ${ }^{5-8}$.

A adicção ao Facebook é um fenômeno recente, não possuindo critérios diagnósticos definidos e encontrando resistência entre alguns pesquisadores ${ }^{8}$. No entanto, alguns países, principalmente os asiáticos, vêm considerando as adições tecnológicas (internet, videogames, redes sociais) um problema de saúde e promovendo a abertura de centros de tratamentos especializados para o transtorno?.

A adicção ao Facebook compartilha características comuns, com outros quadros aditivos [dependência química (DQ) e jogo patológico (JP)] ${ }^{6}$, tais como saliência, modificação do humor, tolerância, abstinência, recaídas e conflitos interpessoais e intrapsíquicos ${ }^{10,11}$. Para Griffiths, essas características compõem o aspecto central das dependências comportamentais ${ }^{10,12}$. Além dessas características, estudos neuropsicológicos e de neuroimagem estrutural e funcional têm encontrado similaridades entre $\mathrm{DQ}, \mathrm{JP}$ e a adicção à internet ${ }^{13}$, da qual a adicção ao Facebook pode ser considerado um subtipo ${ }^{14}$.

Do ponto de vista neuropsicológico, estudos têm sustentado que a adicção à internet apresenta redução nos mecanismos de controle dos impulsos do córtex pré-frontal e outras funções executivas similar àquela encontrada em outros transtornos aditivos ${ }^{13}$. Um dos aspectos neuropsicológicos mais estudados é a tomada de decisão. Usuários excessivos de internet, quando avaliados pelo lowa Gambling Task, apresentam pior desempenho, ou seja, pior tomada de decisão ${ }^{15}$. Outro estudo aponta que adictos a jogos pela internet têm mais propensão a riscos e escolhas desvantajosas ${ }^{16}$. Esses aspectos também são vistos em outros transtornos aditivos.

Outras similaridades entre a adicção à internet, particularmente os adictos a jogos pela internet, e outros transtornos aditivos são verificados nos estudos de neuroimagem. Estudo comparando indivíduos que jogavam frequentemente jogos pela internet e aqueles não frequentes encontrou aumento do volume da substância cinzenta da região ventrolateral esquerda do striatum e maior ativação da região ventral do striatum em jogadores frequentes em comparação com os infrequentes. Os autores concluem que essas mudanças na região ventral do striatum podem refletir mudanças no sistema de recompensa cerebra $1^{17}$.

Um estudo realizado com 18 adolescentes adictos à internet verificou diminuição do volume da substância cinzenta em diversas regiões do lobo frontal - córtex pré-frontal dorsolateral, córtex orbitofrontal, área motora suplementar -, bem como em partes posteriores do cérebro - cerebelo e córtex cingulado anterior rostral à esquerda. Os autores concluíram que essas mudanças podem estar associadas com o prejuízo das cognições relacionadas ao controle inibitório e que essas mudanças são similares àquelas encontradas em dependentes químicos ${ }^{18}$. Outros estudos precisam ser feitos dando ênfase às alterações neuropsicológicas e neurobiológicas nos adictos a redes sociais, tendo em vista a ampliação do entendimento desse fenômeno.

Embora a compreensão atual dessa adicção ainda não esteja completa, não é possível ignorar o problema. A demora em reconhecer e avaliar esse fenômeno permitirá a sua silenciosa propagação, afetando milhões de pessoas, especialmente crianças e adolescentes.

Para avaliar a adicção ao Facebook, um instrumento psicométrico foi desenvolvido por pesquisadores noruegueses. A BFAS, composta por 18 itens, os quais investigam as seis dimensões das dependências comportamentais ${ }^{10,12}$, apresentou alfa de Cronbach 0,83, coeficiente de correlação intraclasse $(\mathrm{CCl})$ de 0,82 e estrutura fatorial satisfatória. Foi validada em uma amostra de estudantes universitários, entretanto os autores sugerem que a BFAS pode ser usada tanto em estudos epidemiológicos de base comunitária como em cenários clínicos ${ }^{11}$.

A adicção ao Facebook é um fenômeno que precisa de mais estudos, sobretudo nas populações que apresentam os maiores indicadores de uso dessa RS. Ainda não existe nenhum estudo que tenha investigado ou validado instrumentos psicométricos para rastreio do uso problemático de Facebook pela população brasileira. O primeiro passo para que isso aconteça é a elaboração ou adaptação transcultural (ATC) de um instrumento psicométrico para esse fim. Por isso, o objetivo deste trabalho foi avaliar a equivalência semântica (ES) e a confiabilidade da BFAS traduzida para o português (Brasil).

\section{MÉTODOS}

A ES foi verificada em uma amostra de conveniência de 37 estudantes da Universidade de Pernambuco, dos cursos de Medicina, Enfermagem, Odontologia, Educação Física e Ciências Biológicas, com idade média de 19,49 anos (DP $= \pm 2,3)$, sendo $64,86 \%(n=24)$ do sexo feminino. A confiabilidade foi estimada em uma amostra de 359 estudantes dos mesmos cursos com idade média de 19,55 anos (DP = 
$\pm 2,37)$, sendo $75,5 \%(n=271)$ do sexo feminino. A escolha de realizar o estudo em uma população de estudantes de Saúde foi no intuito de replicar outros estudos de validação da BFAS que usaram amostras de universitários de outras áreas do conhecimento. Embora sempre validado em amostras de universitários, o instrumento também tem sido utilizado em populações adultas.

Para essa análise, utilizou-se a abordagem recomendada por Herdman et al. ${ }^{19}$ e Reichenhein e Moraes ${ }^{20}$, que preconiza uma abordagem universalista para os estudos de ATC. Outros estudos ${ }^{20-22}$ seguiram o mesmo guideline e foram utilizados como referencial metodológico.

O instrumento original em inglês foi inicialmente traduzido para o português (Brasil) por dois tradutores independentes ${ }^{20,21}$, em seguida foi gerada uma versão síntese. Posteriormente, essa versão foi retraduzida para inglês por dois tradutores, sendo um deles cidadão nativo de país língua inglesa; as duas versões foram comparadas pelos tradutores, que procederam à elaboração de uma versão-síntese da retradução.

A versão-síntese da retradução foi submetida à revisão técnica e à análise da ES por dois psicólogos experientes em ATC de instrumentos psicométricos; os profissionais compararam o original em inglês com a versão-síntese retraduzida, enfatizando a transferência do significado referencial e geral do instrumento ${ }^{21}$; nessa etapa o instrumento foi aprimorado e a versão produzida foi submetida à avaliação da compreensão verbal por 10 especialistas em saúde mental (dois psiquiatras, quatro psicólogos e quatro enfermeiras).

A compreensão verbal foi avaliada para cada item por meio de um questionário que perguntava: "Você entendeu o que está sendo perguntado?". As respostas eram do tipo Likert: 0 - não entendi nada; 1 - entendi só um pouco; 2 entendi mais ou menos; 3 - entendi quase tudo, mas tive algumas dúvidas; 4 - entendi quase tudo; 5 - entendi perfeitamente e não tenho dúvidas. Para que o item seja classificado com grau de compreensão adequado, ele deve obter $80 \%$ de respostas 4 e 5 . Para os itens com compreensão verbal insuficiente, foi solicitado aos participantes que sugerissem modificações, as quais deveriam ser justificadas ${ }^{23}$, e por fim foi produzida a versão pré-final.

A versão pré-final foi aplicada a uma amostra de 37 estudantes, com objetivo de avaliar a compreensão verbal, seguindo o mesmo procedimento metodológico realizado com o grupo de 10 profissionais. Após análise das respostas, uma versão final da BFAS-BR foi redigida.

A versão final foi aplicada a uma amostra de 359 estudantes para análise da confiabilidade do instrumento.

O poder discriminativo dos itens indica o grau em que um item distingue indivíduos que obtêm pontuações altas e baixas ${ }^{24}$. A análise do poder discriminativo dos itens foi realizada por meio do coeficiente de correlação total dos itens e da correlação ponto bisserial, que são as medidas da correla- ção entre cada item e o escore total do instrumento ${ }^{25,26}$. Esse tipo de análise serve para a tomada de decisão sobre a permanência ou exclusão de um determinado item, ao apontar o quanto de contribuição o item ofereceu ao seu domínio. Determinou-se 0,30 como valor mínimo para inclusão25.

Para verificar a estabilidade do instrumento no tempo, optou-se pela técnica de teste-reteste. Desse modo, a cada cinco entrevistas iniciais, um sujeito foi convidado a participar de uma nova entrevista, depois de decorridos 15 dias, até que ao menos $20 \%$ da amostra inicial fossem contatados. Ao final, 72 indivíduos compareceram à segunda entrevista. A partir da obtenção do escore total da BFAS-BR em dois momentos distintos no tempo, determinou-se a estabilidade do instrumento por meio do $\mathrm{CCl}$. A reprodutibilidade seria considerada adequada para CCI maior que $0,70^{27}$.

A consistência interna do instrumento foi verificada por meio do alfa de Cronbach, sendo considerados bons indicadores valores variando entre 0,8-0,928.

A análise estatística foi realizada com o software Statistical Package for the Social Sciences (SPSS, v. 20). A correlação ponto bisserial foi estimada pelo software estatístico Winsteps.

\section{Considerações éticas}

Este estudo seguiu a Resolução n 196/96, do Conselho Nacional de Saúde para pesquisa em seres humanos. Todos os participantes assinaram um Termo de Consentimento Livre e Esclarecido (TCLE). O presente estudo foi aprovado pelo Comitê de Ética e Pesquisa da Universidade Federal de São Paulo (Unifesp), sob o CAAE n 10900712.0.0000.5505 e Parecer $n^{\circ} 173.337 / 12$.

\section{RESULTADOS}

\section{Adaptação transcultural}

Durante o processo de tradução e retrotradução, algumas palavras ou expressões necessitaram ser adequadas para garantir a transferência do sentido denotativo e conotativo das dos termos em questão (Tabela 1):

- O termo Facebook ${ }^{\oplus}$, por se tratar de nome próprio, não foi traduzido para o português;

- No item 8, o termo "helplessness", traduzido como "impotência", foi adaptado para a palavra "incapacidade";

- No item 17, a expressão "hobbies" não foi traduzida para o português falado no Brasil, sendo mantido ipsis litteris como na versão original em inglês.

\section{Avaliação do instrumento}

A análise da escala BFAS-BR pelos profissionais de saúde mental revelou valores médios de compreensão verbal, variando entre 4,4 e 5,0 (Tabela 2). Apenas o item 6 não 
demonstrou um bom grau/nível de compreensão verbal, apresentando, pelos profissionais, uma taxa de respostas 4 e 5 inferior a 80\%, portanto, conforme estabelecido na metodologia deste estudo, o item necessitou ser redigido novamente com base nas considerações dos profissionais, na tentativa de garantir bom nível de compreensão verbal. A redação final do item 6 ficou: "Sentiu que precisava usar cada vez mais o Facebook (por mais tempo e/ou mais vezes) para obter o mesmo prazer?".

Após essa alteração, a escala foi aplicada a um grupo de estudantes para verificar os índices de compreensão verbal, que variou entre 4,5 e 5,0 (Tabela 2), tendo alcançado todos os itens um bom grau de compreensão verbal, portanto não necessitando de mais nenhum ajuste semântico.

A análise da confiabilidade por meio da consistência interna revelou alfa de Cronbach para o instrumento de 0,92; já as dimensões apresentaram alfa de Cronbach variando entre 0,74-0,86. Considerando o alfa se algum item fosse deletado, observou-se que não houve nenhum acréscimo no alfa total de escala. A correlação total dos itens do instrumento estava adequada, variando entre 0,47 e 0,91. A correlação ponto bisserial também foi adequada, variando entre 0,47 e 0, 70 (Tabela 3)

Para a estabilidade no tempo, o instrumento apresentou alto grau de concordância com CCl, igual a 0,81.

Tabela 1. Comparação entre a versão original em inglês, tradução, retrotradução e a versão final

\begin{tabular}{|c|c|c|c|}
\hline Original em inglês & Sintese das traduçōes & Sintese das retrotraduçōes & Versão final \\
\hline $\begin{array}{l}\text { 1. Spent a lot of time thinking about } \\
\text { Facebook or planned use of Facebook? }\end{array}$ & $\begin{array}{l}\text { Passou muito tempo pensando no Facebook } \\
\text { ou planejando usá-lo? }\end{array}$ & $\begin{array}{l}\text { Have you spent much time thinking about } \\
\text { Facebook or planning to use it? }\end{array}$ & $\begin{array}{l}\text { Passou muito tempo pensando sobre } 0 \\
\text { Facebook ou planejando usá-lo? }\end{array}$ \\
\hline $\begin{array}{l}\text { 2. Thought about how you could free more } \\
\text { time to spend on Facebook? }\end{array}$ & $\begin{array}{l}\text { Pensou sobre como você poderia fazer para } \\
\text { ter mais tempo para passar no Facebook? }\end{array}$ & $\begin{array}{l}\text { Have you thought about what you could do } \\
\text { to have more time to spend on Facebook? }\end{array}$ & $\begin{array}{l}\text { Pensou sobre como você poderia fazer para } \\
\text { ter mais tempo para passar no Facebook? }\end{array}$ \\
\hline $\begin{array}{l}\text { 3. Thought a lot about what has happened } \\
\text { on Facebook recently? }\end{array}$ & $\begin{array}{l}\text { Pensou muito sobre o que aconteceu no } \\
\text { Facebook recentemente? }\end{array}$ & $\begin{array}{l}\text { Have you thought a lot of what has happened } \\
\text { on Facebook lately? }\end{array}$ & $\begin{array}{l}\text { Pensou muito sobre o que aconteceu no } \\
\text { Facebook recentemente? }\end{array}$ \\
\hline $\begin{array}{l}\text { 4. Spent more time on Facebook than } \\
\text { initially intended? }\end{array}$ & $\begin{array}{l}\text { Passou mais tempo no Facebook do que você } \\
\text { pretendia inicialmente? }\end{array}$ & $\begin{array}{l}\text { Have you spent more time on Facebook than } \\
\text { you originally intended? }\end{array}$ & $\begin{array}{l}\text { Passou mais tempo no Facebook do que você } \\
\text { pretendia inicialmente? }\end{array}$ \\
\hline $\begin{array}{l}\text { 5. Felt an urge to use Facebook more and } \\
\text { more? }\end{array}$ & $\begin{array}{l}\text { Sentiu necessidade de usar o Facebook mais } \\
\text { e mais? }\end{array}$ & $\begin{array}{l}\text { Have you felt the need to use Facebook more } \\
\text { and more? }\end{array}$ & $\begin{array}{l}\text { Sentiu necessidade de usar o Facebook mais } \\
\text { e mais? }\end{array}$ \\
\hline $\begin{array}{l}\text { 6. Felt that you had to use Facebook more } \\
\text { and more in order to get the same } \\
\text { pleasure from it? }\end{array}$ & $\begin{array}{l}\text { Sentiu que precisava usar o Facebook cada } \\
\text { vez mais para obter o mesmo prazer que } \\
\text { antes? }\end{array}$ & $\begin{array}{l}\text { Have you felt the need to use Facebook more } \\
\text { to obtain the same pleasure as before? }\end{array}$ & $\begin{array}{l}\text { Sentiu que precisava usar cada vez mais o } \\
\text { Facebook (por mais tempo e/ou mais vezes) } \\
\text { para obter o mesmo prazer? }\end{array}$ \\
\hline $\begin{array}{l}\text { 7. Used Facebook in order to forget about } \\
\text { personal problems? }\end{array}$ & $\begin{array}{l}\text { Usou o Facebook a fim de esquecer } \\
\text { problemas pessoais? }\end{array}$ & $\begin{array}{l}\text { Have you used Facebook to forget personal } \\
\text { problems? }\end{array}$ & $\begin{array}{l}\text { Usou o Facebook a fim de esquecer } \\
\text { problemas pessoais? }\end{array}$ \\
\hline $\begin{array}{l}\text { 8. Used Facebook to reduce feelings } \\
\text { of guilt, anxiety, helplessness, and } \\
\text { depression? }\end{array}$ & $\begin{array}{l}\text { Usou o Facebook para reduzir sentimentos de } \\
\text { culpa, ansiedade, impotência ou depressão? }\end{array}$ & $\begin{array}{l}\text { Have you used Facebook to reduce feelings of } \\
\text { guilt, anxiety, helplessness or depression? }\end{array}$ & $\begin{array}{l}\text { Usou o Facebook para reduzir sentimentos } \\
\text { de culpa, ansiedade, incapacidade ou } \\
\text { depressão? }\end{array}$ \\
\hline $\begin{array}{l}\text { 9. Used Facebook in order to reduce } \\
\text { restlessness? }\end{array}$ & Usou o Facebook para reduzir inquietações? & Have you used Facebook to reduce anxieties? & Usou o Facebook para reduzir o estresse? \\
\hline $\begin{array}{l}\text { 10. Experienced that others have told you } \\
\text { to reduce your use of Facebook but not } \\
\text { listened to them? }\end{array}$ & $\begin{array}{l}\text { Foi aconselhado por outros a reduzir seu uso } \\
\text { do Facebook, mas não os escutou? }\end{array}$ & $\begin{array}{l}\text { Have you been advised by others to reduce } \\
\text { your use of Facebook but not listened to } \\
\text { them? }\end{array}$ & $\begin{array}{l}\text { Foi aconselhado por amigos e/ou familiares } \\
\text { a reduzir seu uso do Facebook, mas não os } \\
\text { escutou? }\end{array}$ \\
\hline $\begin{array}{l}\text { 11. Tried to cut down on the use of } \\
\text { Facebook without success? }\end{array}$ & $\begin{array}{l}\text { Tentou reduzir } 0 \text { uso do Facebook, mas não } \\
\text { obteve sucesso? }\end{array}$ & $\begin{array}{l}\text { Have you tried to reduce the use of Facebook, } \\
\text { but without success? }\end{array}$ & $\begin{array}{l}\text { Tentou reduzir o uso do Facebook, mas não } \\
\text { obteve sucesso? }\end{array}$ \\
\hline $\begin{array}{l}\text { 12. Decided to use Facebook less frequently, } \\
\text { but not managed to do so? }\end{array}$ & $\begin{array}{l}\text { Decidiu usar o Facebook com menos } \\
\text { frequência, mas não conseguiu diminuir a } \\
\text { frequência? }\end{array}$ & $\begin{array}{l}\text { Have you decided to use Facebook less } \\
\text { frequently, but could not decrease the } \\
\text { frequency? }\end{array}$ & $\begin{array}{l}\text { Decidiu usar o Facebook com menos } \\
\text { frequência, mas não conseguiu? }\end{array}$ \\
\hline $\begin{array}{l}\text { 13. Become restless or troubled if you have } \\
\text { been prohibited from using Facebook? }\end{array}$ & $\begin{array}{l}\text { Ficou inquieto ou incomodado quando foi } \\
\text { proibido de usar o Facebook? }\end{array}$ & $\begin{array}{l}\text { Have you ever become restless or bothered } \\
\text { when you were forbidden to use Facebook? }\end{array}$ & $\begin{array}{l}\text { Ficou inquieto ou incomodado quando foi } \\
\text { proibido de usar o Facebook? }\end{array}$ \\
\hline $\begin{array}{l}\text { 14. Become irritable if you have been } \\
\text { prohibited from using Facebook? }\end{array}$ & $\begin{array}{l}\text { Ficou irritado quando foi proibido de usar } 0 \\
\text { Facebook? }\end{array}$ & $\begin{array}{l}\text { Have you become irritated when you were } \\
\text { forbidden to use Facebook? }\end{array}$ & $\begin{array}{l}\text { Ficou irritado quando foi proibido de usar } 0 \\
\text { Facebook? }\end{array}$ \\
\hline $\begin{array}{l}\text { 15. Felt bad if you, for different reasons, } \\
\text { could not log on to Facebook for some } \\
\text { time? }\end{array}$ & $\begin{array}{l}\text { Sentiu-se mal quando não pôde acessar } 0 \\
\text { Facebook por algum tempo? }\end{array}$ & $\begin{array}{l}\text { Have you felt bad, for different reasons, } \\
\text { when you could not access Facebook for a } \\
\text { while? }\end{array}$ & $\begin{array}{l}\text { Sentiu-se mal quando não pôde acessar } 0 \\
\text { Facebook por algum tempo? }\end{array}$ \\
\hline $\begin{array}{l}\text { 16. Used Facebook so much that it has had a } \\
\text { negative impact on your job/studies? }\end{array}$ & $\begin{array}{l}\text { Usou tanto o Facebook que teve um impacto } \\
\text { negativo nos seus estudos/trabalho? }\end{array}$ & $\begin{array}{l}\text { Have you used Facebook so much that it has } \\
\text { had a negative impact on your studies/work? }\end{array}$ & $\begin{array}{l}\text { Usou tanto o Facebook que teve um impacto } \\
\text { negativo nos seus estudos/trabalho? }\end{array}$ \\
\hline $\begin{array}{l}\text { 17. Given less priority to hobbies, leisure } \\
\text { activities, and exercise because of } \\
\text { Facebook? }\end{array}$ & $\begin{array}{l}\text { Deu menos prioridade aos hobbies, } \\
\text { atividades de lazer e exercícios por causa do } \\
\text { Facebook? }\end{array}$ & $\begin{array}{l}\text { Have you given less priority to hobbies, } \\
\text { leisure activities and exercises because of } \\
\text { Facebook? }\end{array}$ & $\begin{array}{l}\text { Deu menos prioridade aos hobbies, } \\
\text { atividades de lazer e exercícios por causa do } \\
\text { Facebook? }\end{array}$ \\
\hline $\begin{array}{l}\text { 18. Ignored your partner, family members, } \\
\text { or friends because of Facebook? }\end{array}$ & $\begin{array}{l}\text { Ignorou seu/sua parceiro(a), familiares ou } \\
\text { amigos por causa do Facebook? }\end{array}$ & $\begin{array}{l}\text { Have you ignored your partner, family or } \\
\text { friends because of Facebook? }\end{array}$ & $\begin{array}{l}\text { Ignorou seu/sua parceiro(a), familiares ou } \\
\text { amigos por causa do Facebook? }\end{array}$ \\
\hline
\end{tabular}

Dimensões da BFAS-BR: Saliência: 1, 2, 3; Tolerância: 4, 5, 6; Alteração do humor: 7, 8, 9; Recaída: 10, 11, 12; Abstinência: 13, 14, 15; Conflito: 16, 17, 18.

Todas as questões possuíram as mesmas opções de respostas; trata-se de uma escala do tipo Likert, com valores de 1 a 5: (1) Muito raramente, (2) Raramente, (3) Às vezes, (4) Muitas vezes, (5) Muito frequentemente. 
Tabela 2. Avaliação da compreensão verbal

\begin{tabular}{|c|c|c|c|c|}
\hline \multirow[t]{2}{*}{ Itens BFAS-BR } & \multicolumn{2}{|c|}{$\begin{array}{l}\text { Profissionais } \\
(n=10)\end{array}$} & \multicolumn{2}{|c|}{$\begin{array}{c}\text { Estudantes } \\
(\mathrm{n}=37)\end{array}$} \\
\hline & Grau de compreensão Média (DP*) & $\%$ de respostas 4 e 5 profissionais & Grau de compreensão Média (DP*) & $\%$ de respostas $4 \mathrm{e} 5$ estudantes \\
\hline 1 & $4,5(0,85)$ & $80 \%$ & $4,8(0,39)$ & $97,3 \%$ \\
\hline 2 & $4,6(0,84)$ & $80 \%$ & $4,5(1,01)$ & $83,8 \%$ \\
\hline 3 & $4,6(0,84)$ & $80 \%$ & $4,8(0,70)$ & $94,6 \%$ \\
\hline 4 & $5(0)$ & $100 \%$ & $4,9(0,36)$ & $97,3 \%$ \\
\hline 5 & $4,8(0,63)$ & $90 \%$ & $4,8(0,51)$ & $97,3 \%$ \\
\hline 6 & $4,4(0,96)$ & $70 \%$ & $4,5(1,12)$ & $86,5 \%$ \\
\hline 7 & $5(0)$ & $100 \%$ & $4,7(1,15)$ & $94,6 \%$ \\
\hline 8 & $5(0)$ & $100 \%$ & $4,6(1,23)$ & $91,9 \%$ \\
\hline 9 & $4,5(0,85)$ & $80 \%$ & $4,7(1,02)$ & $91,9 \%$ \\
\hline 10 & $5(0)$ & $100 \%$ & $5(0)$ & $100 \%$ \\
\hline 11 & $4,8(0,63)$ & $90 \%$ & $4,8(0,65)$ & $97,3 \%$ \\
\hline 12 & $5(0)$ & $100 \%$ & $4,9(0,16)$ & $100 \%$ \\
\hline 13 & $5(0)$ & $100 \%$ & $4,9(0,49)$ & $97,3 \%$ \\
\hline 14 & $5(0)$ & $100 \%$ & $4,8(0,60)$ & $94,6 \%$ \\
\hline 15 & $4,9(0,31)$ & $100 \%$ & $4,9(0,32)$ & $97,3 \%$ \\
\hline 16 & $5(0)$ & $100 \%$ & $4,9(0,32)$ & $97,3 \%$ \\
\hline 17 & $4,8(0,63)$ & $94,4 \%$ & $4,9(0,16)$ & $100 \%$ \\
\hline 18 & $5(0)$ & $100 \%$ & $4,9(0,49)$ & $100 \%$ \\
\hline Total & $4,8(0,21)$ & & $4,8(0,15)$ & \\
\hline
\end{tabular}

*DP: desvio-padrão.

Tabela 3. Consistência interna, correlação total dos itens e correlação ponto bisserial

\begin{tabular}{|c|c|c|c|}
\hline Itens & $\begin{array}{l}\text { Correlação total } \\
\text { dositens }\end{array}$ & $\begin{array}{c}\text { Correlação } \\
\text { ponto bisserial }\end{array}$ & $\begin{array}{c}\text { Alfa se item } \\
\text { excluído }\end{array}$ \\
\hline Saliência & & a de Cronbach 0,79 & \\
\hline 1 & 0,69 & 0,67 & 0,91 \\
\hline 2 & 0,91 & 0,53 & 0,92 \\
\hline 3 & 0,64 & 0,59 & 0,92 \\
\hline Tolerância & & a de Cronbach 0,77 & \\
\hline 4 & 0,52 & 0,70 & 0,92 \\
\hline 5 & 0,71 & 0,67 & 0,91 \\
\hline 6 & 0,60 & 0,57 & 0,91 \\
\hline Alteraçōes do humor & & a de Cronbach 0,84 & \\
\hline 7 & 0,74 & 0,56 & 0,92 \\
\hline 8 & 0,70 & 0,53 & 0,92 \\
\hline 9 & 0,68 & 0,63 & 0,92 \\
\hline Recaída & & a de Cronbach 0,86 & \\
\hline 10 & 0,62 & 0,53 & 0,91 \\
\hline 11 & 0,83 & 0,60 & 0,91 \\
\hline 12 & 0,79 & 0,60 & 0,91 \\
\hline Abstinência & & a de Cronbach 0,84 & \\
\hline 13 & 0,73 & 0,58 & 0,91 \\
\hline 14 & 0,81 & 0,56 & 0,91 \\
\hline 15 & 0,61 & 0,63 & 0,91 \\
\hline Conflitos & & a de Cronbach 0,74 & \\
\hline 16 & 0,47 & 0,49 & 0,92 \\
\hline 17 & 0,64 & 0,49 & 0,92 \\
\hline 18 & 0,59 & 0,47 & 0,92 \\
\hline a de Cronbach total & & 0,92 & \\
\hline
\end{tabular}

\section{DISCUSSÃO}

O objetivo deste estudo foi verificar a equivalência semântica e estimar os indicadores de confiabilidade da versão em português (Brasil) da BFAS. A versão adaptada para a população brasileira possui equivalência semântica com o instrumento original em inglês e níveis aceitáveis de consistência interna e estabilidade no tempo.

Na etapa de tradução, a palavra "Facebook" não foi traduzida. Um dos tradutores argumentou que, por ser o nome próprio de uma empresa, não haveria tradução; desse modo, optou-se por mantê-lo igual à versão original. Nas demais etapas revisão técnica, análise da equivalência semântica e avaliação da compreensão verbal (profissionais e estudantes), não houve contestação. Embora o termo tenha sido compreendido pela população-alvo, a literatura científica apresenta críticas à manutenção desse termo, argumentando que a BFAS foi desenvolvida para avaliar a adicção a uma companhia comercial particular mais do que a atividade em si (social networking). Por outro lado, argumenta-se que o conceito de sites de RS não é mais específico do que Facebook, assim adicção ao Facebook em vez de adicção à RS é defensável ${ }^{29}$. De qualquer modo, os pesquisadores devem considerar que o Facebook é um entre muitos outros sites de RS, portanto recomendamos cautela para usar a BFAS na avaliação da adicção para outros sites ou redes sociais de maneira mais ampla.

A avaliação do significado geral e referencial foi realizada durante todo o processo de ES, assegurando que os termos 
traduzidos do inglês para o português transmitissem a carga subjetiva esperada, como observado nos itens 8 e 17. Na tradução do item 8, o termo "helplessness" foi traduzido como impotência, no entanto a mera tradução do termo seria incapaz de transferir o significado geral esperado para o termo na população-alvo, tendo em vista que o termo "impotência" tem um significado cultural distinto para a população brasileira, às vezes carregado de preconceito; por isso, optou-se pelo termo sinônimo "incapacidade", o qual apreende o significado esperado para o termo. No item 17, a palavra "hobbies" foi mantida como no original em inglês por dois motivos: (1) a palavra já está incorporada à cultura-alvo, como observado em vários textos jornalísticos, e constitui um estrangeirismo frequente entre a população brasileira; (2) o termo "hobbies" consegue transferir o significado referencial e geral desejado para o item em questão.

A adaptação envolveu a análise da compreensão verbal dos itens por um grupo de profissionais de saúde mental e por estudantes de graduação da área da Saúde. Para o grupo de profissionais, o item 6 apresentou grau de compreensão verbal insuficiente. Após análise das sugestões dos profissionais para esse item, percebeu-se que havia a necessidade de explicitar de alguma forma a dimensão frequência em que o fenômeno ocorre, o que garantiria maior compreensibilidade do que está sendo avaliado. A análise da compreensão verbal feita pelos estudantes, incluindo o item 6 modificado, revelou que a BFAS-BR foi adequadamente compreendida pelos alunos, não havendo mais necessidade de nenhuma adequação na BFAS-BR, o que garante boa compreensão verbal da versão em português do instrumento.

O alfa de Cronbach para o instrumento adaptado foi de 0,92, superior ao verificado por Andreassen et al. ${ }^{11}$. Da mesma forma, as dimensões apresentaram alfa de Cronbach satisfatório, sendo assim os itens estavam consistentemente reunidos para medir suas respectivas dimensões. $\mathrm{O}$ alfa de Cronbach deve variar entre 0,80 e 0,9028, pois valores superiores a 0,90 são sugestivos de redundância ou duplicação no conjunto de itens, isto é, a existência de itens praticamente iguais escritos de forma diferente, o que deve ser verificado a posteriori em estudo das propriedades psicométricas da BFAS-BR.

A análise de discriminação dos itens refere-se à propriedade de que os itens da escala discriminem a amostra em categorias, sejam elas de alta ou baixa e leve, moderada ou alta performance. A capacidade de discriminação de um item é uma das principais características psicométricas relativa à qualidade de uma questão ${ }^{30}$. Dois dos indicadores do poder de discriminação dos itens são a correlação total dos itense a correlação ponto bisserial26,30. Itens com correlações inferiores a 0,3 devem ser excluídos do teste, uma vez que indicam baixo poder discriminativo e repercutem negativamente na consistência interna do teste. A BFAS-BR apresentou todos os itens com correlações item total e ponto bisserial acima de 0,47 , indicando que todas as questões do instrumento contribuem satisfatoriamente para discriminar indivíduos com níveis baixo e alto de adicção ao Facebook.

A estabilidade do instrumento no tempo foi verificada por meio do $\mathrm{CCl}$, que foi de 0,81 , valor próximo ao encontrado no estudo original de Andreassen et al. ${ }^{11}$, que foi de 0,82 . Cabe destacar que neste estudo o intervalo de tempo entre o teste e o reteste foi de duas semanas e no estudo de Andreassen et al."11 o intervalo foi de três semanas. Esses achados sugerem que a BFAS-BR é estável no tempo.

Embora não haja consenso metodológico sobre a ATC de instrumentos psicométricos, as abordagens que consideram a dimensão cultura $1{ }^{20}$ têm recebido atenção da comunidade acadêmica. A equivalência semântica e a mensuração (confiabilidade) são apenas duas das etapas requeridas pela ATC, sendo as únicas realizadas em alguns estudos ${ }^{21,31}$, o que sugere aceitação desse procedimento no campo científico. É importante enfatizar que essas etapas e suas respectivas estratégias devem ser rigorosamente descritas e cumpridas para garantir a veracidade e a qualidade da informação apresentada ${ }^{32}$, o que foi rigorosamente cumprido neste estudo.

\section{CONCLUSÃO}

Conclui-se, com base no rigor metodológico executado na avaliação da equivalência semântica, que a tradução da BFAS para o português (Brasil) conseguiu transferir o significado geral e referencial do instrumento e que apresentou compreensão verbal satisfatória pelos grupos de profissionais e estudantes. Além disso, mostrou-se um instrumento com bons indicadores de confiabilidade. Os itens, de fato, parecem medir o construto adicção ao Facebook e cada uma de suas respectivas dimensões. O instrumento foi estável no tempo.

Este estudo não encerra a adaptação transcultural do instrumento, pois ainda é necessário verificar outras qualidades psicométricas do instrumento. Dessa forma, recomendamos cautela no uso do instrumento; neste ponto ele serve como ferramenta apenas para nortear o uso em indivíduos com adicção ao Facebook.

\section{CONTRIBUIÇÕES INDIVIDUAIS}

Hugo Rafael de Souza e Silva - Contribuiu com a concepção, projeto, análise e interpretação dos dados, redação do artigo e aprovação final da versão a ser publicada.

Dartiu Xavier da Silveira - Contribuiu com a concepção, projeto, análise e interpretação dos dados, redação do artigo e aprovação final da versão a ser publicada.

Kelsy Catherina Nema Areco - Contribuiu com a análise e interpretação dos dados, revisão crítica relevante do conteúdo intelectual e aprovação final da versão a ser publicada. 
Paulo Bandiera-Paiva - Contribuiu com a análise e interpretação dos dados, revisão crítica relevante do conteúdo intelectual e aprovação final da versão a ser publicada.

Pauliana Valéria Machado Galvão - Contribuiu com a análise e interpretação dos dados, revisão crítica relevante do conteúdo intelectual e aprovação final da versão a ser publicada.

Anália Nusya de Medeiros Garcia - Colaborou na concepção e projeto, revisão crítica relevante do conteúdo intelectual e aprovação final da versão a ser publicada.

Todos os autores foram responsáveis por todos os aspectos do trabalho para a garantia da exatidão e da integridade de qualquer parte da obra.

\section{CONFLITOS DE INTERESSE}

Os autores Dartiu Xavier da Silveira, Kelsy Catherina Nema Areco, Paulo Bandiera-Paiva e Anália Nusya de Medeiros Garcia declaram a inexistência de conflito de interesses em relação ao presente artigo. Hugo Rafael de Souza e Silva declara receber bolsa de doutorado da Coordenação de Aperfeiçoamento de Pessoal de Nível Superior (Capes/Ministério da Educação) e Pauliana Valéria Machado Galvão receber bolsa de doutorado da Coordenação de Aperfeiçoamento de Pessoal de Nível Superior (Capes/Ministério da Educação), com vínculo no doutorado de Epidemiologia de Saúde Pública, da Escola Nacional de Saúde Pública Sérgio Arouca, Fundação Oswaldo Cruz.

\section{REFERÊNCIAS}

1. Boyd DM, Ellison NB. Social network sites: definition, history, and scholarship. J Comput Mediat Commun. 2007;13(1):210-30.

2. Carlson N. Facebook has more than 600 million users, Goldman tells clients. Business Insider. 2011;5:2011.

3. Gomes HS. Brasil é $02^{\circ}$ país com mais usuários que entram diariamente no Facebook. Disponível em: <http://g1.globo.com/tecnologia/noticia/2013/09/brasil-e-0-2-pais-commais-usuarios-que-entram-diariamente-no-facebook.html>. Acesso em: 12 dez, 2013.

4. Ventura F. Com 1,19 bilhão de usuários, Facebook está mais forte do que nunca. Disponível em: <http://gizmodo.uol.com.br/facebook-3q2013/>. Acesso em: 12 dez, 2013.

5. Kuss D, Griffiths M. Excessive online social networking: can adolescents become addicted to Facebook. Education and Health. 2011;29(4):68-71.

6. Kuss DJ, Griffiths MD. Online social networking and addiction - a review of the psychological literature. Int J Environ Res Public Health. 2011;8(9):3528-52.

7. Karaiskos D, Tzavellas E, Balta G, Paparrigopoulos T. Social network addiction: a new clinical disorder? Eur Psychiatry. 2010;25:855.
8. Griffiths MD. Facebook addiction: concerns, criticism, and recommendations - a response to Andreassen and colleagues. Psychol Rep. 2012;110(2):518-20.

9. Huang XQ, Li MC, Tao R. Treatment of internet addiction. Curr Psychiatry Rep. 2010;12:462-70.

10. Griffiths M. A components model of addiction within a biopsychosocial framework. J Subst Use. 2005;10(4):191-7.

11. Andreassen CS, Torsheim T, Brunborg GS, Pallesen S. Development of a Facebook addiction scale. Psychol Rep. 2012;110(2):501-17.

12. Griffiths M. Nicotine, tobacco and addiction. Nature. 1996;384(6604):18.

13. Brand M, Young KS, Laier C. Prefrontal control and internet addiction: a theorical model and review of neuropsychological and neuroimaging findings. Front Hum Neurosci. 2014;8(375):1-13

14. Davis RA, Flett GL, Besser A. Validation of a new scale for measuring problematic internet use: implications for pre-employment screening. Cyberpsychol Behav. 2002;5(4):331-45.

15. Sun DL, Chen ZJ, Ma N, Zhang XC, Fu XM, Zhang DR. Decision-making and prepotent response inhibition functions in excessive internet users. CNS Spectr. 2009;14(2):75-81.

16. Pawlikowski M, Brand M. Excessive internet gaming and decision making: do excessive World of Warcraft-players have problems in decision making under risk conditions? Psychiatry Res. 2011;188(3):428-33.

17. Kuhn S, Romanowski A, Schilling C, Lorenz R, Morsen C, Seiferth N, et al. The neural basis of video gaming. TransI Psychiatry. 2011;15:e53.

18. Yuan $\mathrm{K}$, Qin W, Wang G, Zeng F, Zhao L, Yang X, et al. Microstruture abnormalities in adolescents with internet addiction disorder. Plos One. 2011;6(6):e20708.

19. Herdman M, Fox-Rushby J, Badia X. A model of equivalent in the cultural adaptation of HRQoL instruments: the universalist approach. Qual Life Res. 1998;7(4):323-35.

20. Reichenheim M, Moraes C. Operacionalização de adaptação transcultural de instrumentos de aferição usados em epidemiologia. Rev Saúde Pública. 2007;41(4):665-73.

21. Conti M, Jardim A, Hearst N, Cordás T, Tavares H. Avaliação da equivalência semântica e consistência interna de uma versão em português do Internet Addiction Test (IAT). Rev Psiq Clín. 2012;39(3):106-10.

22. Toledo E, Taragano R, Cordás T, Abreu C, Hearst N, Conti M. Adaptação transcultural da Massachusetts General Hospital (MGH) Hairpulling Scale para o idioma português (Brasil). Rev Psiq Clín. 2011;38(5):178-83.

23. Conti M, Latorre M. Estudo de validação e reprodutibilidade de uma escala de silhueta para adolescentes. Psicol Estud. 2009;14(4):699-706.

24. Bisquerra R, Sarriera JC, Martinez F. Introdução à Estatística - enfoque informático com 0 pacote estatístico SPSS. Porto Alegre: Artmed; 2004.

25. Field A. Descobrindo a estatística usando o SPSS. 2a ed. Porto Alegre: Artmed; 2009.

26. Soares TM. Utilização da teoria da resposta ao item na produção de indicadores socioeconômicos. Pesqui Oper. 2005;25(1):83-112.

27. Shrout $P$, Fleiss J. Intraclass correlations: uses in assessing rater reliability. Psychol Bull. 1979;86(2):420-8.

28. Streiner D. Being inconsistent about consistency: when coefficient alpha does and doesn't matter. J Personal Assess. 2003;80(3):217-22.

29. Andreassen C, Pallesen S. Facebook addiction: a reply to Griffiths (2012). Psychol Rep. 2013;113(3):899-902

30. Collares C, Grec W, Machado J. Psicometria na garantia da qualidade da educação médica: conceitos e aplicações. Sci Health. 2012;3(1):33-49.

31. Toledo E, Taragano R, Cordás T, Abreu C, Hearst N, Conti M. Adaptação transcultural da Massachusetts General Hospital (MGH) Hairpulling Scale para o idioma português (Brasil). Rev Psiq Clín. 2011;38(5):178-83.

32. Conti M, Scagliusi F, Queiroz G, Hearst N, Cordás T. Adaptação transcultural: tradução e validação de conteúdo para o idioma português do modelo da Tripartite Influence Scale de insatisfação corporal. Cad Saude Publica. 2010;26(3):503-13. 